\title{
Optical properties of (113) GaAs/AIAs superlattices grown by molecular beam epitaxy and atomic layer molecular beam epitaxy
}

\author{
G. Bacquet, F. Hassen, ${ }^{\text {a) }}$ and N. Lauret \\ Département de physique, INSA, Avenue de Rangueil, 31077 Toulouse Cedex, France \\ G. Armelles, P. S. Dominguez, and L. Gonzalez \\ Centro Nacional de Microelectrónica (CSIC), Serrano 144, E28006 Madrid, Spain
}

(Received 27 June 1994; accepted for publication 27 September 1994)

\begin{abstract}
We have investigated the optical properties of GaAs/AlAs superlattices grown by molecular beam epitaxy (MBE) and atomic layer MBE (ALMBE) on $\{113\} A$-oriented GaAs substrates. The corrugation which appears in the samples obtained by MBE does not exhibit any periodicity. Phonon-associated replicas are only observed on the photoluminescence excitation spectra of ALMBE superlattices. It was found that the observed differences can be attributed to the growth techniques employed in the synthesis of the samples: MBE superlattices have rougher interfaces than the ALMBE ones, but a lower number of nonradiative recombination centers. (C) 1995 American Institute of Physics.
\end{abstract}

\section{INTRODUCTION}

The GaAs/AlAs system is frequently used in the fabrication of artificially layered semiconductor crystals. The improvement of advanced epitaxial growth techniques, such as molecular beam epitaxy (MBE), has made it possible the obtention of high-quality superlattices (SL's). The beststudied cases, both from experimental and theoretical points of view, are the heterostructures grown on the $\{001\} \mathrm{GaAs}$ surface. The growth of high-quality quantum wells (QW's) and/or SL's on non-\{001\} GaAs substrates has not been successfully carried out until very recently. In this context, the $\{113\} A$ GaAs orientation has attracted much attention since the possibility of directly synthesizing quantum wire-like (QWR) structures without pre- or post-growth substrate patterning has been pointed out by Nötzel et al. ${ }^{1}$ According to these authors, at sufficiently high substrate temperatures (around $600^{\circ} \mathrm{C}$ ) the streaks of the $\{113\} A$ surface reflection high-energy electron diffraction (RHEED) pattern corresponding to the $\langle 33 \overline{2}\rangle$ azimuth exhibit a modulation of their intensity that may be associated with the spontaneous formation of a two-level reconstruction of the surface. In their view, the flat $\{113\} A$ surface breaks up into a series of $\{3 \overline{1} 3\} B$ planes (assumed to have lower surface energy), giving rise to a periodic array of channels and ridges, $1.02 \mathrm{~nm}$ high and 3.2 $\mathrm{nm}$ wide, oriented along the $\langle 33 \overline{2}\rangle$ direction. In addition to this, they postulate a peculiar interchange of channel and ridge positions every time a layer of AlAs is deposited by $\mathrm{MBE}$ on this corrugated GaAs surface (and vice versa). Both facts would, in principle, allow for the fabrication of QWRlike structures just by optimizing the thickness of each constituent layer within a GaAs/AlAs SL. On the other hand photoluminescence (PL) and PL excitation (PLE) studies have revealed the existence of optical anisotropies in the $\{113\} A$ oriented GaAs/AlAs SL's grown by MBE. This anisotropy and other optical properties, such as the increased PL intensity, the red shift of the PL peaks, and the appearance of

\footnotetext{
2) Present address: Département de physique, Faculté des Sciences, Route de
} Kairouan, 5000 Monastir, Tunisia. intense phonon related lines in the PL and PLE spectra, were correlated by Nötzel et al. with the presence of a QWR-like structure. ${ }^{1}$ However, as far as the RHEED pattern is concerned, we have not been able to observe such characteristic splitting of RHEED streaks when growth takes place by atomic layer molecular beam epitaxy (ALMBE) ${ }^{2}$ at low substrate temperatures (around $350{ }^{\circ} \mathrm{C}$ ). In addition, it has been recently shown ${ }^{3}$ that optical anisotropy is not a fingerprint of a QWR structure, but can be present also in samples with no QWR-like structure.

In this paper we present a study of the optical properties of several GaAs/AlAs SL's grown by MBE and by ALMBE on $\{113\} A$ oriented GaAs substrates. The GaAs layer thicknesses have becn varied between 5 to $2.9 \mathrm{~nm}$. As an example we present the results of two samples. The GaAs layer thicknesses are $2.9 \mathrm{~nm}$ for sample 1 (grown by $\mathrm{MBE}$ at a substrate temperature of $600^{\circ} \mathrm{C}$ ) and $3.4 \mathrm{~nm}$ for sample 2 (grown by ALMBE at a substrate temperature of $350^{\circ} \mathrm{C}$ ). The AlAs layer thickness was $8.5 \mathrm{~nm}$ for both samples. All our samples were structurally characterized by double-crystal $x$-ray diffraction (DCXRD) and by Raman scattering spectroscopy. The experimental Raman scattering spectra of the SL's studied here did not present the features characteristic of a periodically corrugated interface. ${ }^{4}$

\section{EXPERIMENTAL DETAILS}

To fulfill the optical spin-orientation condition, the PL of the samples, which were mounted free of strain on their holder and immersed in pumped liquid helium, were excited with $\sigma^{+}$or $\sigma^{-}$circularly polarized light from a tunable (DCM or Pyridine 1) dye laser pumped by an $\mathrm{Ar}^{+}$-ion laser. The polarized PL and PLE were detected and analyzed with a HRS2 Jobin-Yvon simple monochromator with a cooled GaAs-cathode photomuliplier using standard lock-in detection. Circularly polarized excitation light was generated using a Glan-Taylor polarizer followed by a quarterwave Fresnel rhomb. Suitable quarter-wave plates followed by a polarizer were introduced into the detection path. The circular degree of polarization of the PL is defined as 
$P=\left(L^{+}-L^{-}\right) /\left(L^{+}+L^{-}\right)$, where $L^{+}$(resp. $\left.L^{-}\right)$is the intensity of the $\sigma^{+}$-polarized (resp. $\sigma^{-}$) component of the PL. Optical anisotropy experiments were carried out with the exciting light linearly polarized parallel to [233] or to [110], the linear polarizer in front of the receiving monochromator being collinear with [233]. As in the case of circular polarization, we define the degree of optical anisotropy as $P^{\prime}=(L[\overline{2} 33]-L[1 \overline{1} 0]) /(L[\overline{2} 33]+L[1 \overline{1} 0])$, where $L[233]$ (resp. $L[110]$ ) is the PL intensity with the polarization of the excitation parallel to [233] (resp. [110]). For the recording of the PLE spectra the detection was generally set on the low-energy side of the PL line under investigation and the excitation power density was controlled and kept constant (typically $0.8 \mathrm{~W} \mathrm{~cm}^{-2}$ ).

\section{RESULTS}

\section{A. Sample 1}

The PL of this sample consists of a line centered at 1.724 $\mathrm{eV}$, with a more or less marked bump on its high-energy side $(\approx 1.734 \mathrm{eV})$. Its full width at half-maximum (FWHM) varies from 28 to $36 \mathrm{meV}$ when the pump intensity increases. Across the line, the values of both $P$ and $P^{\prime}$ remain constant [respectively, $50 \%$ and $6 \%$ for $h \nu_{\text {exc }}$ close to the (1hh-1e) PLE peak]. A weak narrow peak, associated with an increase of $P^{\prime}(7 \% \rightarrow 14 \%)$, and following the laser energy at (36 $\pm 0.5) \mathrm{meV}$ can be observed on the high-energy side of the PL line for convenient excitation energies as shown in Fig. 1(a).

PLE and circularly polarized PLE (PPLE) experiments were carried out with the detection set at various energies on the low-energy side of the PL line without drastic changes on the corresponding spectra. As an example, we present in Fig. 1 (b) the PPLE spectrum recorded with $h \nu_{\text {rec }}=1.7148 \mathrm{eV}$. In such conditions no phonon-related narrow peaks are seen. The measured so-called Stokes' shift amounts to $(47 \pm 4)$ $\mathrm{meV}$. The resolution of the (1hh-1e) and (1lh-1e) peaks at, respectively $(1.771 \pm 0.002) \mathrm{eV}$ and $(1.832 \pm 0.004) \mathrm{eV}$, is relatively poor. A marked dip at $1.858 \mathrm{eV}$ in the $P\left(h \nu_{\text {exc }}\right)$ variation helps us in the attribution of the light-hole transition, even if its position exceeds that of the small peak in the $L^{-}$component of the PPLE spectrum. Important values of $P$ (up to $60 \%$ ) go with the (1hh-1e) transition.

In the optical anisotropy PLE experiments, it is found that with the polarization light aligned parallel to [233], the heavy-hole response is a little more intense compared to the case with the light polarized parallel to [110]. The value of $P^{\prime}$ changes its sign at $1.842 \mathrm{eV}$, an energy which is greater than that of the $(11 \mathrm{~h}-1 e)$ transition, and remains negative up to the maximum exciting energy available with the DCM dye $(2.03 \mathrm{eV})$.

\section{B. Sample 2}

To obtain a signal intensity comparable to that measured with sample 1 , a power density $W_{\text {exc }}$ of $4 \mathrm{~W} \mathrm{~cm}^{-2}$ (instead of $0.8 \mathrm{~W} \mathrm{~cm}^{-2}$ ) is needed.

Analyzing the shape of the PL response, the FWHM of which is $24 \mathrm{meV}$, we note that it is built from two badly resolved lines distant of about $6 \mathrm{meV}$. For a given $h \nu_{\mathrm{exc}}$, the
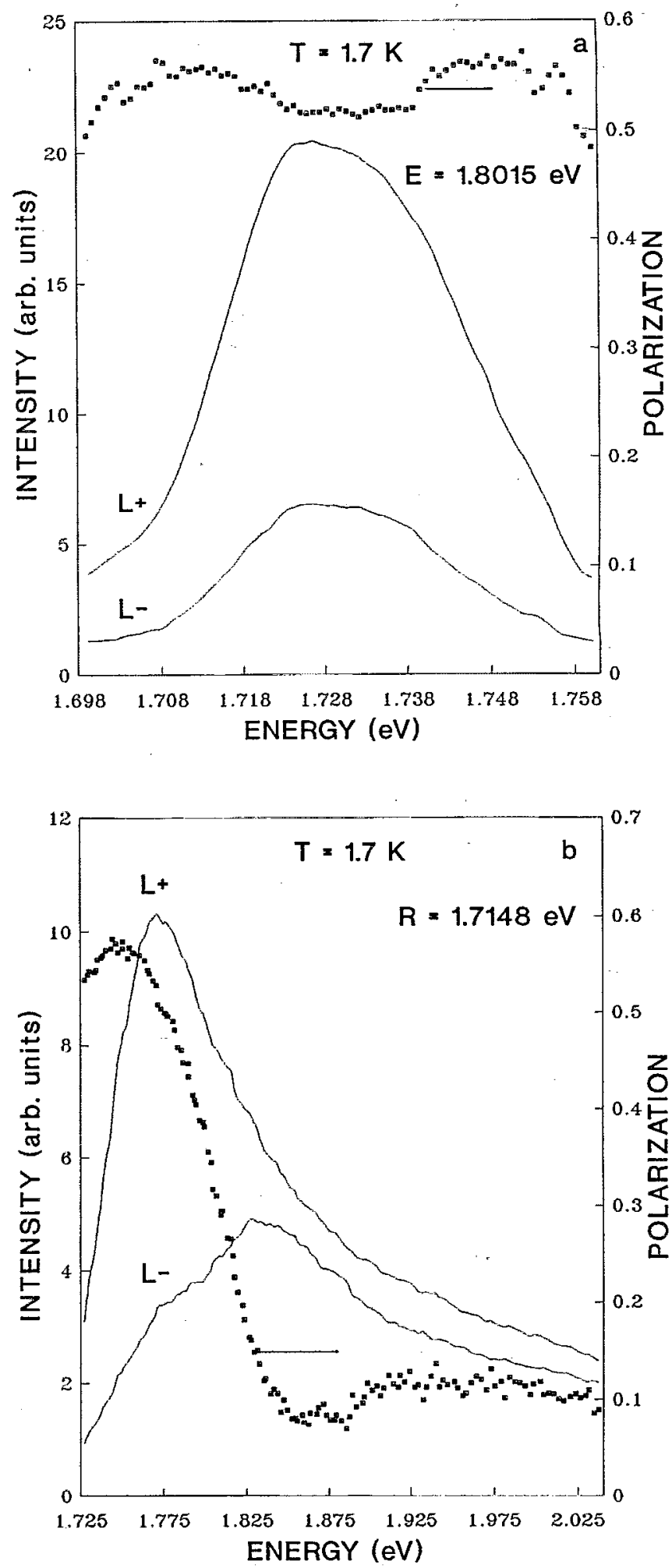

FIG. 1. Sample 1: (a) Polarized PL spectra (left-hand scale) and the corresponding $P\left(h \nu_{\text {rec }}\right)$ variation (right-hand scale). (b) Polarized PLE spectra (left-hand scale) and the corresponding $P\left(h \nu_{\text {exc }}\right)$ variation (right-hand scale).

value of $P$ corresponding to the top of the high-energy line $(1.747 \mathrm{eV})$ is slightly greater than that associated with the top of the low-energy one $(1.741 \mathrm{eV})$ as can be seen in Fig. 2(a). 

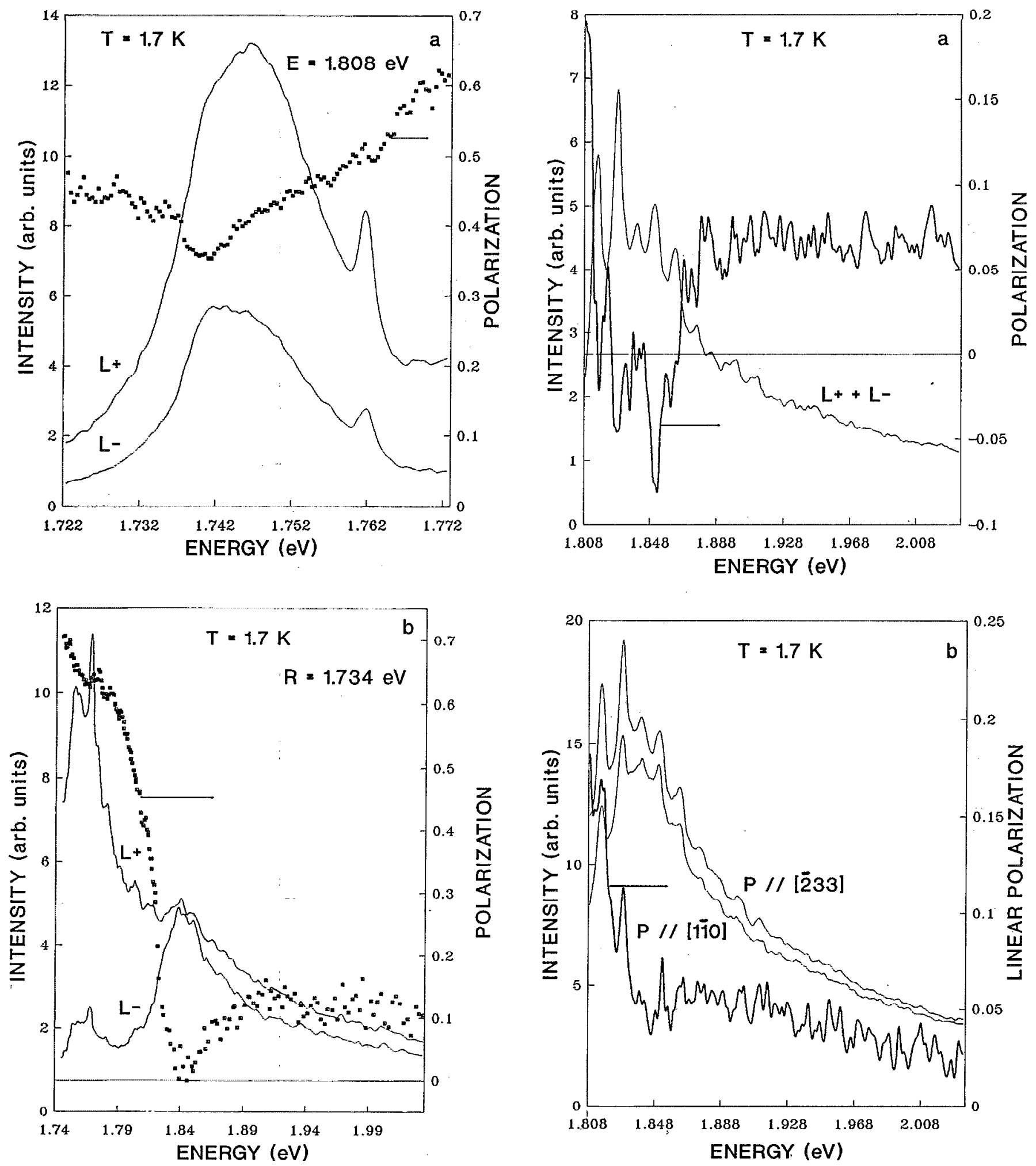

FIG. 2. (a) and (b): The same as in Figs. 1(a) and 1(b) for sample 2.

When the detection is set on the low-energy wing of the $\mathrm{PL}$ response, the (1hh-1e) transition $(1.756 \pm 0.003) \mathrm{eV}$ and the (1lh-1e) one $(1.838 \pm 0.004) \mathrm{eV}$ are clearly observable. The measured Stokes' shift is (12 \pm 3$) \mathrm{meV}$. In Fig. 2(b) is displayed the PPLE spectrum recorded with $h \nu_{\mathrm{rec}}=1.743 \mathrm{eV}$. It can be noted that a strong decrease of the $P\left(\mathrm{~h} \nu_{\mathrm{exc}}\right)$ value corresponds to the $(1 \mathrm{hh}-1 e)$ peak. In the $(1 \mathrm{hh}-1 e)$ region, the

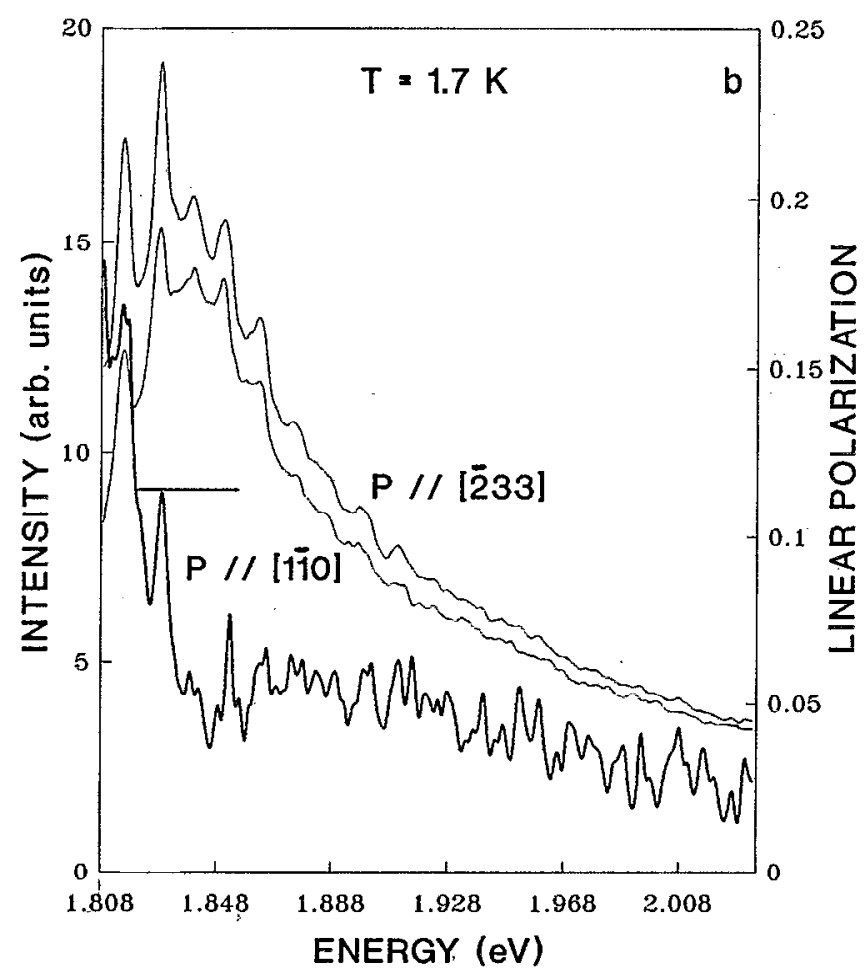

FIG. 3. Sample 2. $h \nu_{\text {rec }}=1.746 \mathrm{eV}$ (high-energy side of the PL line), region of the $(1 \mathrm{lh}-1 e)$ transition where phonon-associated replicas are seen (a) circular polarization, (b) optical anisotropy.

circular degree of polarization amounts to $62 \%$. A narrow doublet, unresolved after the computer treatment of the data, is centered at $h \nu_{\text {rec }}+34.5 \mathrm{meV}$. The same overall spectral features appear in the optical anisotropy PLE spectrum, for which the $P^{\prime}$ value, which always remains positive, is lower 
than that of $P$. However, contrarily to what happens with circularly polarized excitation, the narrow doublet is accompanied by a significative local increase of the $P^{\prime}$ value.

When the detection is set at $1.746 \mathrm{eV}$, seven narrow peaks (or doublets) are observed on the PPLE spectrum, as well as on the optical anisotropy PLE one. They are, respectively, found at $34.5 ; 45 ; 70 ; 83 ; 94 ; 105$, and $116 \mathrm{meV}$ above the detection energy. These seven phonon-related peaks are then associated with a decrease of the $P$ value, especially in the (1lh-1e) region, and an increase of the degree of optical anisotropy as shown in Figs. 3(a) and 3(b) which correspond to this $(1 \mathrm{~h}-1 e)$ region. According to the values reported in Ref. 5 it is possible to attribute these seven phonon-associated peaks respectively to: $1 \mathrm{LO}_{\mathrm{GaAs}}$; $(1 \mathrm{LO}+1 \mathrm{TA})_{\mathrm{GaAs}}$ or $1 \mathrm{LO}_{\mathrm{AIAs}}{ }^{6} 2 \mathrm{LO}_{\mathrm{GaAs}} ;(2 \mathrm{LO}+1 \mathrm{TA})_{\mathrm{GaAs}}$ or $1 \mathrm{LO}_{\mathrm{GaAs}}+1 \mathrm{LO}_{\mathrm{AlAs}} ;(2 \mathrm{LO}+2 \mathrm{TA})_{\mathrm{GaAs}}$ or $2 \mathrm{LO}_{\mathrm{AlAs}} ; 3 \mathrm{LO}_{\mathrm{GaAs}}$ and $(3 \mathrm{LO}+1 \mathrm{TA})_{\mathrm{GaAs}}$.

With an appropriate exciting energy (for instance 1.817 $<h \nu_{\text {exc }}<1.835 \mathrm{eV}$ in the case of the $2 \mathrm{LO}_{\mathrm{GaAs}}$ peak), these phonon-associated features can be seen on the high-energy wing of the PL response with the same behavior concerning the degrees of circular polarization and optical anisotropy as in the PLE spectra.

\section{DISCUSSION}

The results presented here are more or less common to all the other samples and can be summarized as follows. The PL intensity of MBE samples is always greater than that of the ALMBE samples. The Stokes' shift is also greater in the MBE samples. The PLE spectra of ALMBE samples have normally phonon-associated peaks.

The differences in the optical properties of the samples studied can be attributed to the growth techniques employed in their synthesis. The layer by layer nature of ALMBE favors the formation of sharp interfaces, compared with those obtained by MBE. In this way, the somewhat more pronounced corrugation of the MBE sample interfaces can explain the larger Stokes' shift observed in it. This is due to the fact that excitons can be easily trapped in the wider regions of the QW's. Yet it must be emphasized that, according to the Raman measurements, this corrugation does not exhibit any periodicity, as suggested by Nötzel et al. ${ }^{1}$

Nevertheless, ALMBE may induce the creation of a large number of nonradiative recombination centers. The ideal unreconstructed surface of a $\{001\}$ GaAs substrate consists only of either As or $\mathrm{Ga}$ atoms, while the surface of a $\{113\}$ (A or B) GaAs substrate is terminated with both $\mathrm{Ga}$ and As atoms. Thus, in the second case, a rapid and drastic shift in the stoichiometry of the atomic or molecular beams impinging on the surface (ALMBE) may give rise to the incorporation of As atoms into sites of the Ga sublattice, and vice versa. A different number of nonradiative recombination centers can account for the observed differences in the PL intensity (the PL intensity of the MBE sample was always greater than that of the ALMBE one) and also for the existence of phonon-related replicas in the PLE spectra of the ALMBE sample. The presence of nonradiative centers shortens the exciton lifetime. According to Ref. 7, an increase in the exciton lifetime induces a decrease in the intensity of the phonon-associated replicas in the PLE. We would like to point out that we did not observe such phonon replicas in the PLE spectra of the MBE sample, contrary to the findings of Nötzel et al. ${ }^{1}$

In conclusion, we have investigated the optical properties of $\{113\} A$ GaAs/AlAs MQW structures grown by two different methods. According to the results, the MBE sample has rougher interfaces than the ALMBE sample, but a lower number of nonradiative recombination centers.

\section{ACKNOWLEDGMENTS}

G.A., P.S.D., and L.G. wish to thank the Spanish Comision Inteministerial de Ciencia y Tecnologia for financial support (Project No. MAT92-0262).

${ }^{1}$ R. Nötzel, N. N. Ledentsov, L. Däweritz, M. Hohenstein, and K. Ploog, Phys. Rev. Lett. 67, 3812 (1991); R. Nötzel, N. N. Ledentsov, L. Däweritz, K. Ploog, and M. Hohenstein, Phys. Rev. B 45, 3507 (1992).

${ }^{2}$ F. Briones, L. Gonzälez, and A. Ruiz, Appl. Phys. A 49, 729 (1989).

${ }^{3}$ G. Armelles, P. Castrillo, P. S. Domínguez, L. González, A. Ruiz, D. A. Contreras-Solorio, V. R. Velasco, and F. García-Moliner, Phys. Rev. B 49, 14020 (1994).

${ }^{4}$ P. Castrillo, G. Armelles, L. Gonzalez, P. S. Dominguez, and L. Colombo, Phys. Rev. B (in press).

${ }^{5}$ Landolt-Börnstein, Numerical Data and Fundamental Relationships in Sciences and Technology, Group III (Springer, Berlin, 1982), Vol. 17.

${ }^{6}$ D. S. Jiang, X. Q. Thou, M. Oestreich, W. W. Rühle, R. Nötzel, and K. Ploog, Phys. Rev. 49, 10786 (1994).

${ }^{7}$ S. Permogorov, Phys. Status Solidi B 68, 9 (1975) 